\title{
TRANSPIRAÇÃO E TEMPERATURA FOLIAR DA CANA-DE-AÇÚCAR SOB DIFERENTES VALORES DE POTENCIAL MATRICIAL ${ }^{1}$
}

\author{
ROBERTO TRENTIN ${ }^{2}$, SÉRGIO ZOLNIER ${ }^{3}$, ARISTIDES RIBEIRO $^{3}$, \\ ANTONIO J. STEIDLE NETO ${ }^{4}$
}

\begin{abstract}
RESUMO: O objetivo deste trabalho foi avaliar a variação diurna da transpiração e da temperatura foliar da cana-de-açúcar, cv. RB867515, sob diferentes potenciais matriciais de água no substrato de cultivo e as condições meteorológicas em ambiente protegido. $\mathrm{O}$ efeito do estresse hídrico na transpiração e temperatura foliar foi determinado a partir da suspensão da irrigação, quando foram realizadas três campanhas de medições, iniciadas aos 122; 150 e 185 dias após o plantio (DAP) até que o potencial matricial de água no substrato $(\Psi)$ alcançasse $-1.500 \mathrm{kPa}$, aproximadamente. Sob ausência de estresse hídrico $(\Psi>-50 \mathrm{kPa})$, a transpiração das plantas atingiu o valor máximo entre 10 e 13 h, próximo de 60; 70 e $100 \mathrm{~g}$ planta $^{-1} \mathrm{~h}^{-1}$ para 122; 150 e $185 \mathrm{DAP}$, respectivamente. Sob condições de estresse hídrico severo $(\Psi<-1.100 \mathrm{kPa})$, houve redução na transpiração diária de aproximadamente $73 \%$, comparativamente às medições realizadas sem estresse e sob condições meteorológicas similares. Em condições de ausência de estresse hídrico e de céu nublado, o valor médio da diferença entre a temperatura foliar e a temperatura do ar foi de $-2,9{ }^{\circ} \mathrm{C}$. Em contraste, sob condições de estresse hídrico severo e valores elevados de radiação solar global, a temperatura foliar chegou a ser $6,6^{\circ} \mathrm{C}$ superior à temperatura do ar.
\end{abstract}

PALAVRAS-CHAVE: Saccharum officinarum, evapotranspiração, termometria a infravermelho, estresse hídrico, irrigação.

\section{TRANSPIRATION AND LEAF TEMPERATURE OF SUGARCANE UNDER DIFFERENT MATRIC POTENTIAL VALUES}

\begin{abstract}
The objective of this study was to evaluate the diurnal behaviour of transpiration and leaf temperature of sugarcane (cv. RB867515) under different water matrix potential in the cultivation substrate and greenhouse meteorological conditions. The water stress effect on transpiration and leaf temperature was determined after irrigation suspension, when three measurement campaigns were initiated at 122; 150 and 185 days after planting (DAP) until the matrix water potential in the substrate $(\Psi)$ reached $-1,500 \mathrm{kPa}$, approximately. Under the absence of water stress $(\Psi>-50 \mathrm{kPa}$ ), plant transpiration reached the maximum value between 10:00 AM and 13:00 PM, near to 60; 70 e $100 \mathrm{~g} \mathrm{plant}^{-1} \mathrm{~h}^{-1}$, for 122; 150 and 185 DAP, respectively. Under severe water stress $(\Psi<-1,100 \mathrm{kPa})$, there was daily transpiration reduction of approximately $73 \%$, compared to measurements performed without water stress and under similar meteorological conditions. Under the absence of water stress and overcast sky conditions, the average value of the difference between leaf temperature and air temperature was $-2.9^{\circ} \mathrm{C}$. In contrast, under severe water stress and high values of global solar radiation, the leaf temperature was found to be $6.6{ }^{\circ} \mathrm{C}$ above the air temperature.
\end{abstract}

KEYWORDS: Saccharum officinarum, evapotranspiration, infrared thermometry, water stress, irrigation.

\footnotetext{
${ }^{1}$ Extraído da Dissertação de Mestrado do primeiro autor. Bolsa de estudos concedida pelo CNPq e auxílio financeiro para execução do projeto de pesquisa proveniente da FAPEMIG.

${ }^{2}$ Eng ${ }^{\mathrm{O}}$ Agrônomo, Doutorando em Engenharia Agrícola, Departamento de Fitotecnia, CCR/UFSM, Santa Maria - RS, Fone: (0XX55) 3220.8902, robertotrentin@yahoo.com.br.

${ }^{3}$ Eng $^{\mathrm{o}}$ Agrícola, Prof. Doutor, Departamento de Engenharia Agrícola, DEA/UFV, Viçosa - MG.

${ }^{4}$ Eng $^{\mathrm{O}}$ Agrícola, Prof. Doutor, Departamento de BioEngenharia, USL/UFSJ, Campus de Sete Lagoas - MG.

Recebido pelo Conselho Editorial em: 5-11-2010

Aprovado pelo Conselho Editorial em: 2-8-2011
} 


\section{INTRODUÇÃO}

A cana-de-açúcar destaca-se como uma das principais culturas de interesse econômico para o Brasil, fornecendo matéria-prima para a produção de açúcar e álcool, além de atuar como componente da alimentação animal, principalmente na estação seca, e de ser fonte de energia para a geração de energia elétrica a partir do bagaço proveniente da moagem (DALRI \& CRUZ, 2008).

De acordo com SMIT \& SINGELS (2006), o estresse hídrico afeta o desenvolvimento da arquitetura do dossel vegetativo da cana-de-açúcar, o qual é fundamental na produtividade da cultura, uma vez que o dossel intercepta a radiação solar que, por sua vez, atua nos processos de fotossíntese e transpiração. Assim, a condutância estomática da cana-de-açúcar responde às variações de disponibilidade de água no meio de cultivo, de maneira a regular as trocas gasosas de dióxido de carbono e vapor d'água entre a cultura e o ambiente. Dados obtidos por INMANBAMBER (2004) evidenciaram que, se ocorrer estresse hídrico durante o desenvolvimento da cultura da cana-de-açúcar, haverá restrição em processos fisiológicos, como o de divisão celular, causando diminuição na taxa de acúmulo de matéria seca, na taxa de elongação dos colmos e no índice de área foliar. Além disso, com a intensificação do estresse, ocorrem impactos na produtividade da cultura e na concentração de sacarose nos colmos.

As necessidades hídricas da cana-de-açúcar são alteradas ao longo das fases de desenvolvimento. Dependendo da severidade do estresse, da cultivar e da fase fenológica, as plantas tendem a fechar os estômatos, enrolar as folhas e realizar ajustes osmóticos, como mecanismos de tolerância à diminuição da água disponível no meio de cultivo (INMAN-BAMBER \& SMITH, 2005).

O estresse hídrico é uma situação que ocorre com frequência durante o cultivo da cana-deaçúcar em condições de sequeiro. Desta forma, o conhecimento da resposta da planta à disponibilidade hídrica é imprescindível para a seleção de cultivares apropriadas para condições climáticas específicas e adoção de práticas de manejo da cultura, visando a maximizar o uso eficiente de água (SMIT \& SINGELS, 2006).

Em muitas regiões do mundo, incluindo locais classificados climaticamente como úmidos, a cana-de-açúcar é cultivada com a utilização de irrigação ao longo do ciclo da cultura ou, eventualmente, com irrigações suplementares (LEBOURGEOIS et al., 2010). Assim, decisões sobre a frequência e duração dos eventos de irrigação são tomadas por meio de métodos baseados no balanço de água e medições do potencial de água nas folhas, condutância estomática, umidade e potencial de água no solo e temperatura foliar. Nesse sentido, o emprego de sistemas de controle automáticos dos eventos de irrigação, que considerem as variáveis associadas ao ambiente de cultivo e à cultura, assume importância no crescimento e desenvolvimento das plantas (STEIDLE NETO et al., 2009).

A transpiração das plantas é um componente do balanço de energia que determina a temperatura foliar, de acordo com fatores anatômicos das folhas (dimensões, pigmentação e massa), fatores do ambiente (radiação solar, velocidade do ar, temperatura e umidade relativa do ar) e fatores biológicos que determinam o número e a distribuição dos estômatos (MONTEITH \& UNSWORTH, 1990; LEUZINGER et al., 2010). À medida que a água setorna limitante, a transpiração é reduzida, ocorrendo o aumento da temperatura foliar pela absorção da radiação solar incidente (EMEKLI et al., 2007; GONTIA \& TIWARI, 2008; WANG \& GARTUNG, 2010).

Diversos índices de estresse hídrico foram desenvolvidos e continuam sendo aprimorados a partir do conhecimento da resposta das plantas ao potencial de água no sistema radicular, a partir de medições e/ou estimativas de transpiração e temperatura foliar. Muitos índices utilizam a diferença entre a temperatura da cultura $\left(\mathrm{t}_{\mathrm{c}}\right)$ e temperatura do ar $\left(\mathrm{t}_{\mathrm{ar}}\right)$ como base para a estimativa da severidade do estresse hídrico (TESTI et al., 2008; LEBOURGEOIS et al., 2010). Em decorrência da dificuldade de se determinar com exatidão os valores críticos do diferencial " $t_{c}-t_{a r}$ " em condições de campo, especialmente sob condições de estresse hídrico severo, métodos alternativos de estimativa têm sido propostos para obtenção desses valores de referência (WIDMOSER, 2010). 
Este trabalho teve por objetivo avaliar o comportamento diurno da transpiração e da temperatura foliar da cana-de-açúcar em casa de vegetação, sob diferentes tensões matriciais de água no substrato de cultivo e condições meteorológicas, visando a contribuir para o aprimoramento de métodos de estimativa de estresse hídrico em condições de sequeiro e controle da frequência de aplicação de água em plantios irrigados a partir da termometria a infravermelho.

\section{MATERIAL E MÉTODOS}

Para o controle da disponibilidade de água no meio de cultivo, este experimento foi conduzido no interior de um ambiente protegido, pertencente ao Departamento de Engenharia Agrícola da Universidade Federal de Viçosa (DEA/UFV), Viçosa - MG. As coordenadas geográficas do local são: latitude $20^{\circ} 45^{\prime} 45^{\prime}$ S S, longitude $42^{\circ} 52^{\prime} 04^{\prime \prime}$ W e altitude de $690 \mathrm{~m}$.

O ambiente protegido possuía a maior dimensão orientada na direção Leste-Oeste e cobertura construída com estrutura metálica em forma de arco, tendo $15 \mathrm{~m}$ de comprimento, $7 \mathrm{~m}$ de largura e altura central de $3,5 \mathrm{~m}$. O material plástico da cobertura era constituído de filme de polietileno de baixa densidade (PEBD), com espessura de $150 \mu \mathrm{m}$, possuindo aditivos contra a ação de raios ultravioleta. Nas laterais, foi utilizada uma tela de plástico branca, com malha de $1 \mathrm{~mm}$, para reduzir a entrada de insetos.

Para a obtenção das respostas da cana-de-açúcar às diferentes condições meteorológicas e alterações do potencial matricial de água no sistema radicular, foi utilizada a cv. RB867515, que é a mais difundida no Brasil tanto para a produção de açúcar e álcool quanto de forragem (BARBOSA \& SILVEIRA, 2010). Os toletes foram plantados no dia 6-5-2009, em vasos de plástico com capacidade para 10 litros, os quais foram preenchidos com substrato comercial (Bioplant, Nova Ponte, MG) à base de casca de pínus, vermiculita, fibra de coco e complementos minerais (NPK) e micronutrientes. A massa de substrato foi idêntica para todas as plantas cultivadas e, para facilitar a realização das práticas culturais, os vasos também foram dispostos na direção leste-oeste. No total, 192 plantas foram cultivadas durante o período de seis meses, mas apenas três plantas foram utilizadas em cada campanha de medição da evapotranspiração e temperatura foliar.

No fundo de cada vaso, foi perfurado um orifício circular para permitir a drenagem dos excessos de água e da solução nutritiva proveniente da irrigação e fertirrigação, respectivamente. Acima desse orifício, foi colocado um disco de tela de plástico com malha de $1 \mathrm{~mm}$ para impedir a remoção do substrato. Sobre a tela, foram depositados $500 \mathrm{~g}$ de carvão vegetal, visando a facilitar a drenagem lateral na parte inferior do vaso de cultivo.

Durante o período de crescimento, os perfilhos da cana-de-açúcar foram removidos periodicamente, mantendo-se apenas um colmo em cada vaso. As plantas foram irrigadas por meio de microtubos flexíveis que derivavam de tubulações de PVC (Figura 1A). A duração e a frequência dos eventos de irrigação foram controladas por um temporizador. As plantas foram tutoradas individualmente para garantir o crescimento vertical e evitar o tombamento causado pelo próprio peso. Para minimizar a perda de água por evaporação para o ambiente de cultivo, foi colocado um filme de polietileno transparente sobre a superfície do substrato.

Perfis hidropônicos de polipropileno (Hidrogood, Taboão da Serra-SP), com 2,6 m de comprimento, foram utilizados como calhas para a coleta da solução nutritiva drenada em cada fileira, sendo instalados com inclinação de 4\%. Na extremidade mais baixa das calhas, foi instalado um dreno, conectado a um recipiente de quatro litros, para o armazenamento do excedente dos eventos de irrigação ou fertirrigação (Figura 1B). Cada calha coletava a drenagem proveniente de seis vasos de cultivo, havendo no total 32 calhas de coleta de drenagem para os 192 vasos com plantas. 

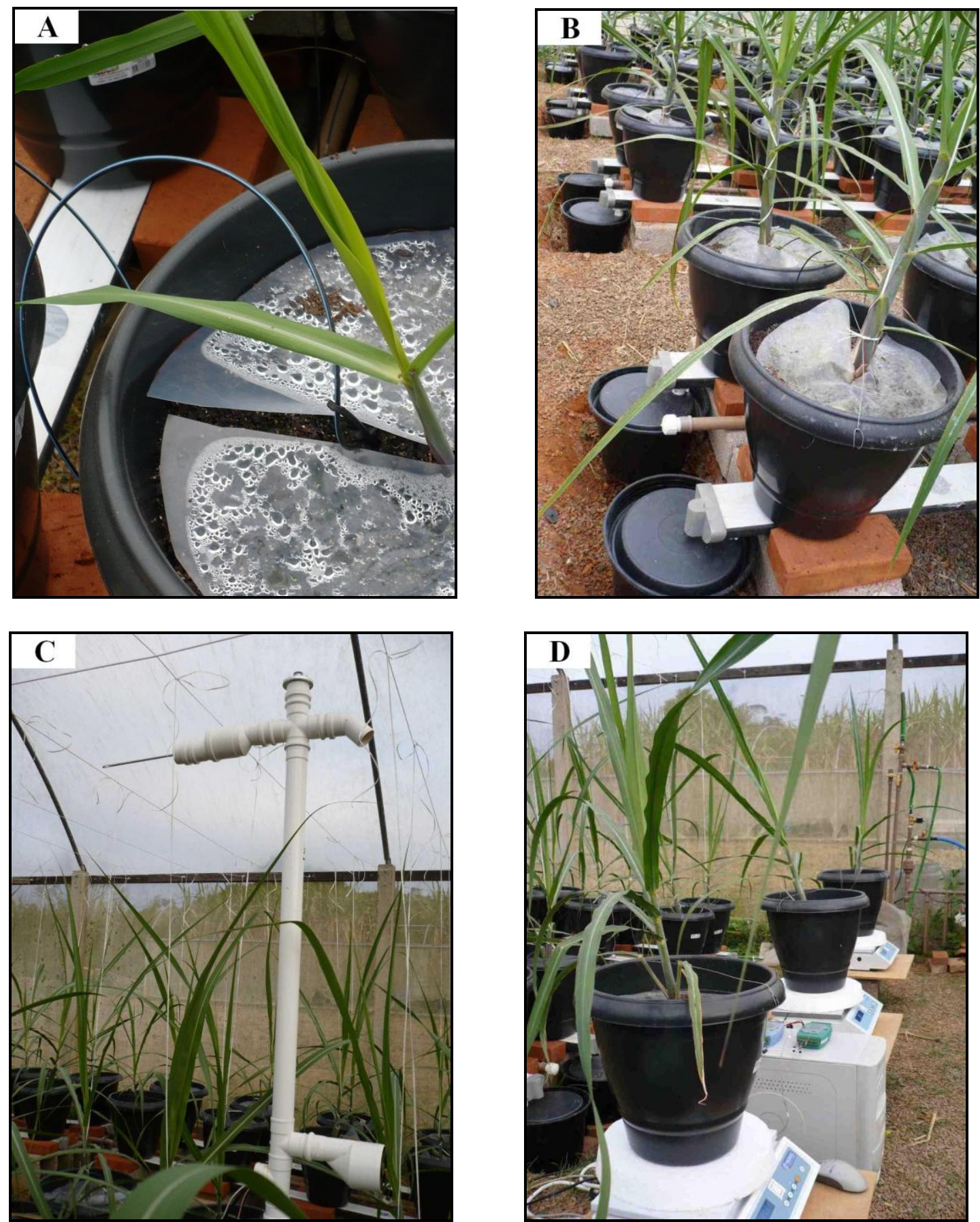

FIGURA 1. Cultivo da cana-de-açúcar sob condições de ambiente protegido (DEA/UFV, Viçosa-MG). As fotos ilustram os sistemas de fertirrigação (A) e de drenagem da solução nutritiva (B), o tubo de PVC com aspiração para instalação dos sensores eletrônicos (C) e o posicionamento das balanças de precisão (D). Sugarcane cultivation under greenhouse conditions (DEA/UFV, Viçosa-MG). The pictures illustrate the fertirrigation (A) and the drainage systems of the nutrient solution (B), the aspirated PVC tube for installing the electronic sensors $(C)$ and the positioning of the precision scales $(D)$.

A condutividade elétrica (CE) da solução percolada no substrato foi monitorada por meio de um condutivímetro (modelo CDH-42, Omega, Stamford, CT, USA), sendo mantida entre 2,0 e $3,0 \mathrm{dSm}^{-1}$ por meio de fertirrigações periódicas. A solução nutritiva foi preparada manualmente em um reservatório de plástico de 100 litros, utilizando-se de um fertilizante solúvel em água (Peters Professional 20-20-20 GP, Marysville, OH, USA). 
A temperatura e a umidade relativa do ar foram medidas por meio de um sensor conjugado (modelo Humitter 50Y, Vaisala, Woburn, MA, USA) que foi instalado no interior de um tubo de PVC com aspiração. A radiação solar global foi obtida por meio de um piranômetro (modelo LI200SA, LI-COR, Lincoln, NE, USA), o qual também foi fixado na torre de aspiração de PVC (Figura 1C).

Os sensores responsáveis pela medição das variáveis meteorológicas no interior do ambiente protegido foram conectados a uma placa de aquisição de dados (modelo CYDAS 1602HR, CyberResearch, Branford, CT, USA), instalada em um microcomputador. Para o gerenciamento do processo de aquisição e armazenamento dos dados provenientes dos sensores, foi desenvolvido um programa computacional em linguagem $C++$ Builder (versão 6.0).

A transpiração foi determinada por meio de três balanças de precisão (Tecnal, Piracicaba-SP), com capacidade individual para $12 \mathrm{~kg}$ e precisão de $0,1 \mathrm{~g}$ (Figura 1D). O sistema foi conectado a um microcomputador por meio de portas seriais. A variação de massa do conjunto, constituído de vaso de plástico, substrato, água e uma planta de cana-de-açúcar, foi medida em intervalos de tempo de 1 min e, posteriormente, integrada para períodos horários.

O efeito do estresse hídrico na transpiração e temperatura foliar foi determinado a partir da suspensão da irrigação em datas distintas, quando foram realizadas três campanhas de medições, iniciadas aos 122; 150 e 185 dias após o plantio (DAP), até que o potencial matricial no substrato

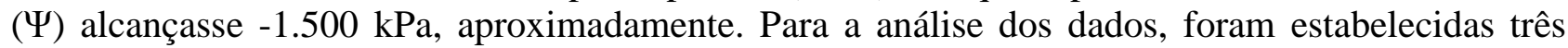
classes de potencial de água: ausência de estresse hídrico $(\Psi>-50 \mathrm{kPa})$, estresse hídrico moderado $(-700<\Psi<-400 \mathrm{kPa})$ e severo $(-1.500<\Psi<-1.100 \mathrm{kPa})$. O experimento foi conduzido em delineamento completamente casualizado, com nove repetições, representadas por plantas individuais, posicionadas sobre três balanças de precisão, e a realização de três campanhas de medições.

Ao final de cada campanha, as plantas foram descartadas, pois o potencial de água no substrato estava próximo do ponto de murcha permanente, e a maior parte da área foliar já estava totalmente desidratada. Assim, em cada período de medição, compreendendo também três classes de estresse hídrico, as quais constituíram os tratamentos, foram utilizadas três plantas que não tinham experimentado, previamente, situações de estresse hídrico, nas quais foram medidas a transpiração, a temperatura foliar e a área foliar. Como os dados do ambiente são específicos em cada campanha de medição, as análises da variação diurna da transpiração e da temperatura foliar da cana-de-açúcar foram realizadas separadamente.

A temperatura foliar foi obtida a partir do posicionamento de um termômetro a infravermelho (modelo 4000.4GL, Everest Interscience, Tucson, AZ, USA), em pontos aleatórios de cada uma das três plantas utilizadas nas medições de transpiração, a uma distância de aproximadamente $2,5 \mathrm{~cm}$, em intervalos de tempo de $1 \mathrm{~h}$. Os pontos de amostragem situavam-se nas folhas superiores, que estavam totalmente abertas, procurando-se apontar o sensor na posição central da superfície foliar.

Para a caracterização física e hídrica do substrato, foram determinadas a densidade aparente e a capacidade de retenção de água do substrato, sendo esta obtida por meio da curva de retenção, utilizando-se de câmaras de pressão de Richards. Durante o período experimental, o potencial matricial de água no substrato de cultivo $(\Psi)$ foi obtido a partir do método proposto por GENUCHTEN (1980) para a estimativa da curva de retenção de água, utilizando-se de valores do conteúdo atual de umidade volumétrica.

Com o objetivo de acompanhar a expansão da área foliar da cana-de-açúcar ao longo do período experimental, foi realizado um monitoramento biométrico uma vez por semana. Para a determinação da área foliar (AF), foram mensurados o comprimento e a largura da porção mediana de todas as folhas de cada planta utilizada nas medições de transpiração e temperatura foliar. Para o cálculo da área foliar, foi adotada a Equação 1, adaptada de HERMANN \& CÂMARA (1999): 


$$
\mathrm{AF}=\sum_{\mathrm{NF}}(\mathrm{C} \text { L } 0,69)
$$

em que,

$\mathrm{AF}$ - área foliar de cada planta amostrada, $\mathrm{cm}^{2}$;

NF - número de folhas;

C - comprimento de cada folha, $\mathrm{cm}$;

L - largura de cada folha, $\mathrm{cm}$, e

0,69 - fator de "forma".

SILVA (2009), conduzindo experimento durante o ciclo de cana-soca, com a cv. RB92579 sob condições do Semiárido Brasileiro, obteve experimentalmente o valor de 0,69 como o mais adequado para realizar essa correção.

\section{RESULTADOS E DISCUSSÃO}

Na Figura 2, estão representadas graficamente a variação horária da transpiração e a da temperatura foliar da cana-de-açúcar no período diurno, sob ausência de estresse hídrico, aos 122; 150 e 185 dias após o plantio (DAP). Adicionalmente, são apresentadas as respectivas áreas foliares (AF) e a variação da temperatura do ar $\left(t_{a r}\right)$, radiação solar global incidente $\left(\mathrm{R}_{\mathrm{g}}\right)$ e déficit de pressão de saturação de vapor d'água no ar $\left(\mathrm{DPV}_{\mathrm{ar}}\right.$, sendo que o último termo foi obtido a partir de equações psicrométricas (VIANELLO \& ALVES, 1991).

Quando totalizada ao longo do período diurno, a transpiração foi de 349; 444 e $423 \mathrm{~g} \mathrm{planta}^{-1} \mathrm{~d}^{-1}$ para $122 ; 150$ e 185 DAP, respectivamente. Observou-se a influência marcante da radiação solar incidente sobre a transpiração, a qual variou de acordo com a nebulosidade nos três dias de medição. Nota-se também que a transpiração aumentou até período compreendido entre $10 \mathrm{e}$ $13 \mathrm{~h}$, com os valores máximos próximos de 60; 70 e $100 \mathrm{~g}_{\text {planta }}{ }^{-1} \mathrm{~h}^{-1}$, respectivamente, para os três dias mencionados. Nos horários subsequentes, houve redução até o término do período diurno. ALVES (1999), conduzindo experimento sem restrição hídrica com a variedade de cana-de-açúcar NA72 454, relatou valores de transpiração máxima entre 40 e $105 \mathrm{~g} \mathrm{planta}^{-1} \mathrm{~h}^{-1}$ sob $\mathrm{R}_{\mathrm{g}}$ de aproximadamente $550 \mathrm{Wm}^{-2}$.

O processo de transpiração está associado à diferença das temperaturas folha-ar " $t_{c}-t_{a r}$ ", sendo que este diferencial se torna mais negativo à medida que ocorre transferência de energia da folha para o ar na forma de calor latente. Sob menores valores de $\mathrm{DPV}_{\mathrm{ar}}$, constatados no período da manhã, a temperatura foliar esteve próxima da temperatura do ar, ocorrendo alternância de valores positivos e negativos do diferencial " $\mathrm{t}_{\mathrm{c}}-\mathrm{t}_{\mathrm{ar}}$ ", destacadamente aos 150 DAP.

Com valores do $\mathrm{DPV}_{\mathrm{ar}}$ superiores aos observados no período da manhã, a temperatura foliar esteve, na maior parte da tarde, abaixo da temperatura do ar nos três dias de medição. Em condições de céu nublado, observadas no período compreendido entre 12 e $16 \mathrm{~h}$ aos $122 \mathrm{DAP}$, a $\mathrm{R}_{\mathrm{g}}$ e o DPV ar estiveram, respectivamente, entre 200 e $400 \mathrm{Wm}^{-2}$ e 13 e $16 \mathrm{hPa}$. Neste período, o valor médio do diferencial " $\mathrm{t}_{\mathrm{c}}-\mathrm{t}_{\mathrm{ar}}$ " foi de $-2,9^{\circ} \mathrm{C}$.

Em contraste, sob condições de céu claro, LEBOURGEOIS et al. (2010) encontraram a relação " $t_{c}-t_{a r}=2,33-0,177 \mathrm{DPV}_{\mathrm{ar}}$ " para a cultivar de cana-de-açúcar $\mathrm{R} 570$, a qual resulta no valor médio de $-0,3{ }^{\circ} \mathrm{C}$ para o diferencial " $\mathrm{t}_{\mathrm{c}}-\mathrm{t}_{\mathrm{ar}}$ " sob níveis de $\mathrm{DPV}$ ar próximos de $15 \mathrm{hPa}$. Portanto, sob ausência de estresse hídrico, observa-se que a $R_{g}$ tende a aumentar o diferencial " $t_{c}-t_{a r}$ ", enquanto o $\mathrm{DPV}_{\mathrm{ar}}$ exerce um efeito oposto. Resultados similares foram relatados por EMEKLI et al. (2007) para a grama bermuda, TESTI et al.(2008) para a pistácia, GONTIA \& TIWARI (2008) para o trigo e WANG \& GARTUNG (2010) para o pessegueiro. 

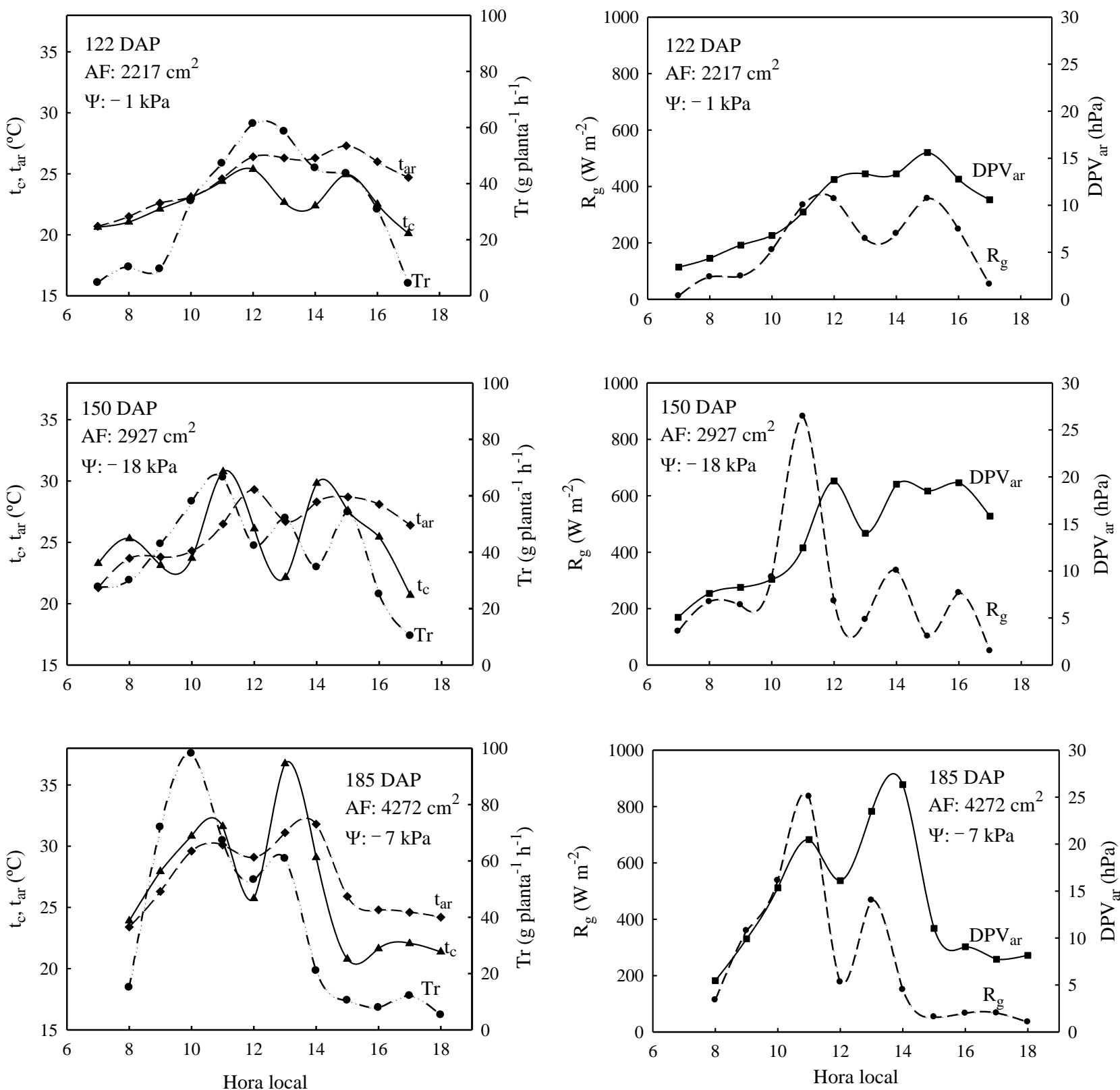

FIGURA 2. Variações diurnas da temperatura foliar $\left(t_{c}\right)$ e transpiração $(T r)$ da cana-de-açúcar (cultivar RB867515), da temperatura do ar $\left(\mathrm{t}_{\mathrm{ar}}\right)$, da radiação solar incidente $\left(\mathrm{R}_{\mathrm{g}}\right)$ e do déficit de pressão de saturação de vapor d'água no ar (DPV $\left.{ }_{\text {ar }}\right)$ para 122; 150 e 185 dias após o plantio (DAP), sob ausência de estresse hídrico. Os símbolos AF e $\Psi$ representam, respectivamente, a área foliar e o potencial matricial de água no substrato. Diurnal variations of the leaf temperature $\left(t_{c}\right)$ and transpiration $(\mathbf{T r})$ of sugarcane (cultivar $\mathbf{R B 8 6 7 5 1 5}$ ), air temperature $\left(t_{a r}\right)$, incident solar radiation $\left(\mathbf{R}_{\mathrm{g}}\right)$ and air vapor pressure deficit $\left(\mathrm{DPV}_{\mathrm{ar}}\right)$ for 122,150 and 185 days after planting (DAP), under the absence of water stress. The symbols AF and $\Psi$ represent respectively the leaf area and the substrate water matrix potencial.

Sob estresse hídrico moderado, aos 126; 154 e 187 DAP (Figura 3), mesmo com o aumento da área foliar, os valores de transpiração diária foram inferiores aos medidos na avaliação sem restrição hídrica, sendo de 251; 108 e 163 gplanta $^{-1} \mathrm{~d}^{-1}$ para 126; 154 e 187 DAP, respectivamente. Observa-se que a transpiração aumentou até atingir valores máximos entre 10 e $12 \mathrm{~h}$, próximos de 40; 20 e $35 \mathrm{~g}_{\text {planta }}{ }^{-1} \mathrm{~h}^{-1}$ para os três DAPs especificados, respectivamente. 
Para níveis de $\Psi$ situados entre -700 e $-400 \mathrm{kPa}$, a menor disponibilidade de água reduziu a transpiração das plantas. Em decorrência disso, a temperatura foliar foi, em geral, superior à temperatura do ar, sendo também afetada pelas oscilações na radiação solar global. No período da manhã, sob valores de $\mathrm{R}_{\mathrm{g}}$ acima de $200 \mathrm{Wm}^{-2}$ e $\mathrm{DPV}_{\mathrm{ar}}$ inferiores a $15 \mathrm{hPa}$, a $\mathrm{t}_{\mathrm{c}}$ esteve, na maior parte do tempo, acima da $t_{a r}$. Em contraste, com valores mais elevados do DPV ar no período da tarde e redução da $R_{g}$ a partir das $14 h$, a $t_{c}$ apresentou valores ligeiramente inferiores em relação a $t_{a r}$.
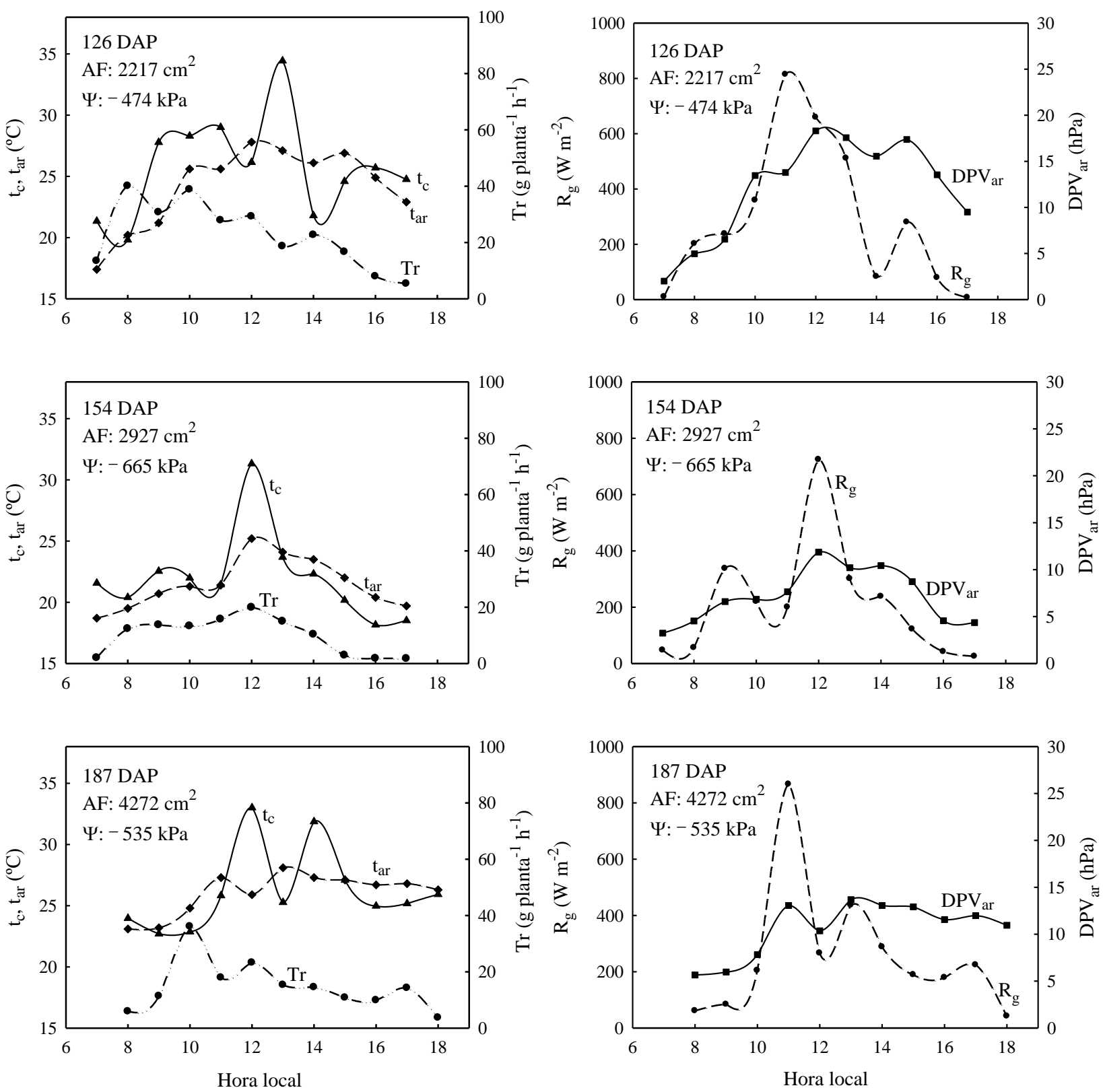

FIGURA 3. Variações diurnas da temperatura foliar $\left(\mathrm{t}_{\mathrm{c}}\right)$ e transpiração $(\mathrm{Tr})$ da cana-de-açúcar (cultivar RB867515), da temperatura do ar $\left(\mathrm{t}_{\mathrm{ar}}\right)$, da radiação solar incidente $\left(\mathrm{R}_{\mathrm{g}}\right)$ e do déficit de pressão de saturação de vapor d'água no ar (DPV $\left.{ }_{a r}\right)$ para 126; 154 e 187 dias após o plantio (DAP), sob condições de estresse hídrico moderado. Os símbolos $\mathrm{AF}$ e $\Psi$ representam, respectivamente, a área foliar e o potencial matricial de água no substrato. Diurnal variations of the leaf temperature $\left(t_{c}\right)$ and transpiration $(\mathbf{T r})$ for sugarcane (cultivar $\mathbf{R B 8 6 7 5 1 5}$ ), air temperature $\left(t_{a r}\right)$, incident solar radiation $\left(R_{g}\right)$ and air vapor pressure deficit $\left(D P V_{a r}\right)$ for 126, 154 and 187 days after planting (DAP), under conditions of moderate water stress. The symbols AF and $\Psi$ represent respectively the leaf area and the substrate water matrix potencial. 
Para condições caracterizadas por estresse hídrico severo aos 128; 156 e 191 DAP (Figura 4), verificou-se comportamento similar ao constatado em condições de estresse hídrico moderado, mas com maior diminuição da transpiração diária, mesmo com o aumento da área foliar.

Verificou-se também que a intensidade da radiação solar global $\left(\mathrm{R}_{\mathrm{g}}\right)$ variou muito no decorrer desses três dias devido à nebulosidade local. Sob níveis de potencial matricial no substrato inferiores a $-1.100 \mathrm{kPa}$, a disponibilidade de água para as plantas tornou-se extremamente limitante. Os valores encontrados para a transpiração foram 128; 114 e 113 gplanta $^{-1} \mathrm{~d}^{-1}$ para 128; 156 e 191 DAP, respectivamente.
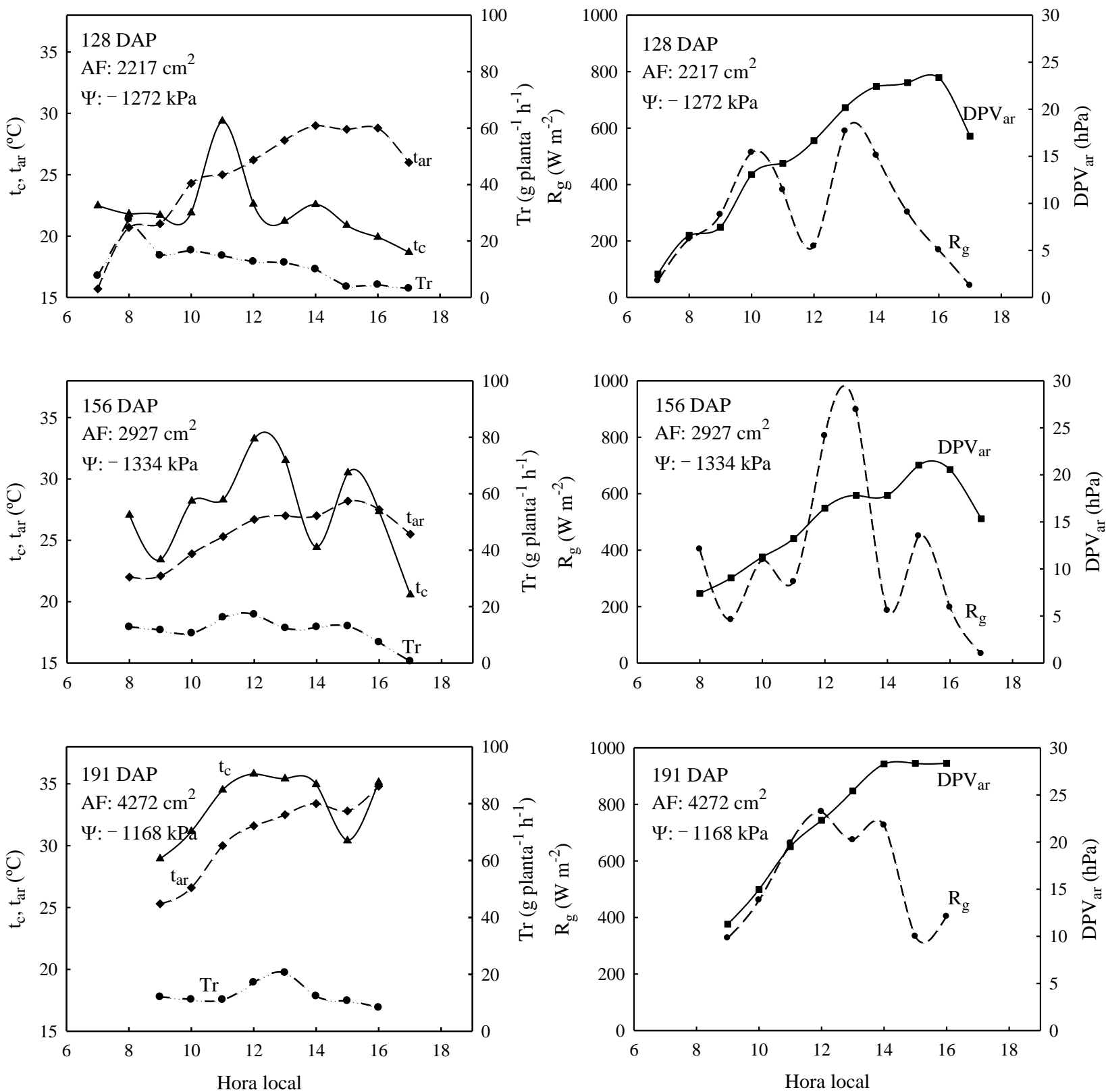

FIGURA 4. Variações diurnas da temperatura foliar $\left(\mathrm{t}_{\mathrm{c}}\right)$ e transpiração $(\mathrm{Tr})$ da cana-de-açúcar (cultivar $\mathrm{RB} 867515)$, da temperatura do ar $\left(\mathrm{t}_{\mathrm{ar}}\right)$, da radiação solar incidente $\left(\mathrm{R}_{\mathrm{g}}\right)$ e do déficit de pressão de saturação de vapor d'água no ar $\left(\mathrm{DPV}_{\mathrm{ar}}\right)$ para 128; 156 e 191 dias após o plantio (DAP), sob estresse hídrico severo. Os símbolos AF e $\Psi$ representam, respectivamente, a área foliar e o potencial matricial de água no substrato. Diurnal variations of the leaf temperature $\left(t_{c}\right)$ and transpiration (Tr) for sugarcane (cultivar RB867515), air temperature $\left(t_{a r}\right)$, incident solar radiation $\left(R_{q}\right)$ and vapor pressure deficit $\left(D P V_{a r}\right)$ for 128, 156 and 191 days after planting (DAP), under severe water stress. The symbols AF and $\Psi$ represent respectively the leaf area and the substrate water matrix potencial. 
Esses resultados são notoriamente inferiores aos medidos nas avaliações sem restrição hídrica e com estresse moderado. Comparado com a avaliação sem estresse hídrico, as reduções na transpiração diária foram de $63 ; 74$ e $73 \%$, respectivamente. Verificam-se, também, reduções drásticas nas taxas de transpiração horária, as quais atingiram, de maneira aproximada, valores máximos de apenas 28; 18 e 20 gplanta $^{-1} \mathrm{~h}^{-1}$ para 128; 156 e $191 \mathrm{DAP}$, respectivamente. No período da tarde, os valores apresentaram queda, prosseguindo assim até o final do período diurno. Segundo SILVA (2009), sob condições de baixa disponibilidade de água nas imediações do sistema radicular, as plantas de cana-de-açúcar tendem a fechar os estômatos, visando a reduzir a perda de água, resultando em maior transferência de calor sensível da superfície da cultura para a atmosfera.

Com o aumento da área foliar em condições de estresse hídrico severo, observado aos 156 e 191 DAP, a $t_{c}$ esteve bem acima da $t_{a r}$, mesmo sob valores elevados do DPV $\mathrm{ar}_{\mathrm{ar}}$. Somente às $14 \mathrm{e} 15 \mathrm{~h}$, respectivamente, aos 156 e 191 DAP, o diferencial " $t_{c}-t_{a r}$ " foi negativo em decorrência da queda acentuada da $\mathrm{R}_{\mathrm{g}}$. Por outro lado, o valor máximo do diferencial " $\mathrm{t}_{\mathrm{c}}-\mathrm{t}_{\mathrm{ar}}$ " foi de $+6,6{ }^{\circ} \mathrm{C}$, o qual foi constatado às $12 \mathrm{~h}$ aos $156 \mathrm{DAP}$, sob valores de $\mathrm{R}_{\mathrm{g}}$ e $\mathrm{DPV}_{\mathrm{ar}}$ de $800 \mathrm{Wm}^{-2}$ e $16 \mathrm{hPa}$, respectivamente.

\section{CONCLUSÕES}

Independentemente da área foliar e sob condições meteorológicas similares, caracterizadas pelos valores máximos de radiação solar global e de déficit de saturação de pressão de vapor d'água no ar, a taxa de transpiração diária da cana-de-açúcar, em condições de estresse hídrico severo $(-1.500<\Psi<-1.100 \mathrm{kPa})$, foi, aproximadamente, $73 \%$ inferior em relação à das plantas cultivadas sob plena disponibilidade de água.

$\mathrm{Na}$ ausência de estresse hídrico, a temperatura foliar acompanhou as oscilações da radiação solar global, causadas por condições de céu parcialmente nublado. No entanto, em condições de céu completamente nublado, observadas no período compreendido entre 12 e 16h, o valor médio do diferencial " $\mathrm{t}_{\mathrm{c}}-\mathrm{t}_{\mathrm{ar}}$ " foi de $-2,9{ }^{\circ} \mathrm{C}$. Em contraste, sob condições de estresse hídrico severo e valores elevados de radiação solar global, a temperatura foliar atingiu até $6,6^{\circ} \mathrm{C}$ acima da temperatura do ar. Esses valores estabelecem condições de contorno para a temperatura foliar da cultura e são imprescindíveis para o desenvolvimento de índices de estresse hídrico que poderão ser utilizados em modelos de estimativa de produtividade e no manejo de irrigação da cana-de-açúcar.

\section{AGRADECIMENTOS}

Esta pesquisa foi realizada com o apoio financeiro da Fundação de Amparo à Pesquisa do Estado de Minas Gerais - FAPEMIG, e do Conselho Nacional de Desenvolvimento Científico e Tecnológico - CNPq. O primeiro autor também agradece à Universidade Federal de Viçosa, por meio do Programa de Pós-Graduação em Meteorologia Agrícola, pela oportunidade para a obtenção do título de Mestre.

\section{REFERÊNCIAS}

ALVES, E. L. Desenvolvimento de um sistema de medição dos fluxos de transpiração líquida para a cultura de cana-de-açúcar a partir do fluxo de seiva. 1999. 71 f. Dissertação (Mestrado em Engenharia Agrícola) - Universidade Estadual de Campinas, Campinas, 1999.

BARBOSA, M.H.P.; SILVEIRA, L.C.I. Melhoramento genético e recomendação de cultivares. In: SANTOS, F.; BORÉM, A.; CALDAS, C. Cana-de-açúcar: bionergia, açúcar e álcool - tecnologias e perspectivas. Viçosa: Universidade Federal de Viçosa, 2010. p.313-331.

DALRI, A.B.; CRUZ, R.L. Produtividade da cana-de-açúcar fertirrigada com N e K via gotejamento subsuperficial. Engenharia Agrícola, Jaboticabal, v.28, n.3, p.516-524, 2008.

EMEKLI, Y.; BASTUG, R.; BUYUKTAS, D.; EMEKLI, N.Y. Evaluation of a crop water stress index for irrigation scheduling of bermudagrass. Agricultural Water Management, Amsterdam, v.90, p.205-212, 2007. 
GENUCHTEN, M. T. van. A closed-form equation for predicting the hydraulic conductivity of unsaturated soils. Soil Science Society America Journal, Madison, v.44, p.892-898, 1980.

GONTIA, N.K.; TIWARI, K.N. Development of crop water stress index of wheat crop for scheduling irrigation using infrared thermometry. Agricultural Water Management, Amsterdam, v.95, p.1.144-1.152, 2008.

HERMANN, E.R.; CÂMARA, G.M.S. Um método simples para estimar a área foliar de cana-de-açúcar. STAB Açúcar, Álcool e Subprodutos, Piracicaba, v.17, n.5, p.32-34, 1999.

INMAN-BAMBER, N.G. Sugarcane water stress criteria for irrigation and drying off. Field Crops Research, Amsterdam, v.89, p.107-122, 2004.

INMAN-BAMBER, N.G.; SMITH, D.M. Water relations in sugarcane and response to water deficits. Field Crops Research, Amsterdam, v.92, p.185-202, 2005.

LEBOURGEOIS, V.; CHOPART, J.L.; BÉGUÉ, A.; Le MÉZO, L. Towards using a thermal infrared index combined with water balance modelling to monitor sugarcane irrigation in a tropical environment. Agricultural Water Management, Amsterdam, v.97, p.75-82, 2010.

LEUZINGER, S.; VOGT, R.; KÖRNER, C. Tree surface temperature in an urban environment. Agricultural and Forest Meteorology, Amsterdam, v.150, p.56-62, 2010.

MONTEITH, J.; UNSWORTH, M. Principles of environmental physics. Londres: Edward Arnold, 1990. $291 \mathrm{p}$.

SILVA, T.G.F. Análise de crescimento, interação biosfera-atmosfera e eficiência do uso de água da cana-de-açúcar irrigada no semiárido brasileiro. 2009. $168 \mathrm{f}$. Tese (Doutorado em Meteorologia Agrícola) - Universidade Federal de Viçosa, Viçosa, 2009.

SMIT, M.A.; SINGELS, A. The response of sugarcane canopy development to water stress. Field Crops Research, Amsterdam, v.98, p.91-97, 2006.

STEIDLE NETO, A.J.; ZOLNIER, S.; MAROUELLI, W.A.; MARTINEZ, H.E.P. Avaliação do desempenho de um sistema automático para controle da fertirrigação do tomateiro cultivado em substrato. Engenharia Agrícola, Jaboticabal, v.29, n.3, p.380-389, 2009.

TESTI, L.; GOLDHAMER, D.A.; INIESTA, F. Crop water stress index is a sensitive water stress indicator in pistachio trees. Irrigation Science, New York, v.26, p.395-405, 2008.

VIANELLO, R.L.; ALVES, A.R. Meteorologia básica e aplicações. Viçosa-MG: Imprensa Universitária, 1991. 448 p.

WANG, D.; GARTUNG, J. Infrared canopy temperature of early-ripening peach trees under postharvest deficit irrigation. Agricultural Water Management, Amsterdam, v.97, p.1.787-1.794, 2010.

WIDMOSER, P. An alternative to define canopy surface temperature bounds. Agricultural Water Management, Amsterdam, v.97, p.224-230, 2010. 